\title{
LP Well-Posedness for Bilevel Vector Equilibrium and Optimization Problems with Equilibrium Constraints
}

\author{
Phan Quoc Khanh, ${ }^{1}$ Somyot Plubtieng, ${ }^{2}$ and Kamonrat Sombut ${ }^{2}$ \\ ${ }^{1}$ Department of Mathematics, International University of Ho Chi Minh City, Linh Trung, Thu Duc, Ho Chi Minh City, Vietnam \\ ${ }^{2}$ Department of Mathematics, Faculty of Science, Naresuan University, Phitsanulok 65000, Thailand \\ Correspondence should be addressed to Somyot Plubtieng; somyotp@nu.ac.th
}

Received 21 January 2014; Accepted 9 March 2014; Published 17 April 2014

Academic Editor: Chong Li

Copyright (C) 2014 Phan Quoc Khanh et al. This is an open access article distributed under the Creative Commons Attribution License, which permits unrestricted use, distribution, and reproduction in any medium, provided the original work is properly cited.

\begin{abstract}
The purpose of this paper is introduce several types of Levitin-Polyak well-posedness for bilevel vector equilibrium and optimization problems with equilibrium constraints. Base on criterion and characterizations for these types of Levitin-Polyak well-posedness we argue on diameters and Kuratowski's, Hausdorff's, or Istrătescus measures of noncompactness of approximate solution sets under suitable conditions, and we prove the Levitin-Polyak well-posedness for bilevel vector equilibrium and optimization problems with equilibrium constraints. Obtain a gap function for bilevel vector equilibrium problems with equilibrium constraints using the nonlinear scalarization function and consider relations between these types of LP well-posedness for bilevel vector optimization problems with equilibrium constraints and these types of Levitin-Polyak well-posedness for bilevel vector equilibrium problems with equilibrium constraints under suitable conditions; we prove the Levitin-Polyak well-posedness for bilevel equilibrium and optimization problems with equilibrium constraints.
\end{abstract}

\section{Introduction}

Well-posedness is one of most important topics for optimization theory and numerical methods of optimization problems, which guarantees that, for approximating solution sequences, there is a subsequence which converges to a solution. The well-posedness of unconstrained and constrained scalar optimization problems was first introduced and studied by Tikhonov [1] and Levitin and Polyak [2], respectively. In Tikhonov well-posedness, which means the existence and uniqueness of minimizer and the convergence of a subsequence of each approximation sequence to a solution, the Tikhonov notation has been more interested; that is, any algorithm can generate only an approximating sequence of solutions. Hence, this sequence is applicable only if the problem under consideration is well posed. The concept of Tikhonov well-posedness has also been generalized to several related problems: variational inequalities, Nash equilibrium problems, optimization problems with variational inequalities constrains, optimization problems with Nash equilibrium constrains, optimization problems with Nash equilibrium constrains [3-12], and so forth.

The study of Levitin-Polyak (LP for short) well-posedness for scalar convex optimization problems with functional constraints originates from [13]. Recently, this research was extended to nonconvex optimization problems with abstract and functional constraints [14] and nonconvex vector optimization problems with both abstract and functional constraints [15]. In 2009, S. J. Li and M. H. Li [16] introduced and researched two types of LP well-posedness of vector equilibrium problems with variable domination structures. In the same year, Huang et al. [17] introduced and researched the LP well-posedness of vector quasi-equilibrium problems. Moreover, Li et al. [18] introduced and researched the LP well-posedness for two types of generalized vector quasiequilibrium problems.

Most recently, many papers appeared dealing with bilevel problems such as mathematical programming with equilibrium constraints $[19,20]$, optimization problems with Nash equilibrium constraints [21], optimization problems 
with variational inequality constraints [4], and optimization problems with equilibrium constraints [20,22]. In 2012, Anh et al. [23] considered the LP well-posedness of bilevel equilibrium problems with equilibrium constraints (BEPEC) and bilevel optimization problems with equilibrium constraints (BOPEC). They introduced a relaxed level closedness and use it together with pseudomonotonic assumptions to establish sufficient conditions of LP well-posedness.

The vector equilibrium problem is a unified model of several classes of problems, for example, vector optimization problems and vector variational inequality problems [24, 25]. In recent years, many authors have intensively studied different types of vector equilibrium problem [26-28]. Many results on existence and stability of solutions for vector equilibrium problem, generalized vector equilibrium problems, and generalized quasivector equilibrium problems have been established [26-31]. Moreover, many authors have investigated the gap functions for vector equilibrium problems, generalized vector equilibrium problems, and generalized quasivector equilibrium problems [28].

Motivated and inspired by the above observations, our consideration of LP well-posedness for bilevel problems is in this paper. We focus on vector equilibria with equilibrium constraints and optimization with equilibrium constraints, as well as an abstract set constraint, and investigate criteria and characterizations for these types of LP well-posedness with a gap function for bilevel vector equilibrium problems with equilibrium constraints and optimization problems with equilibrium constraints. We propose a generalized level closedness and use it together to study well-posedness in the LP sense. However, since the existence topic has been intensively studied for vector equilibrium and bilevel problems, we focus on LP well-posedness, assuming always that the mentioned solutions exist.

The layout of the paper is as follows. In Section 2, we state the bilevel problems under our consideration and recall notions and preliminaries needed in the sequel. In Section 3, we study LP well-posedness of bilevel vector equilibrium problems with equilibrium constraints and optimization problems with equilibrium constraints on diameters and measures of noncompactness of approximate solution sets in the Kuratowski, Hausdorff, or Istrătescu sense. In Section 4, by virtue of a nonlinear scalarization function and a gap function for bilevel vector quasi-equilibrium problems, we show equivalent relations between the LP well-posedness of the optimization problem and the LP well-posedness of bilevel vector equilibrium problems. The results in this paper unify, generalize, and extend some known results in $[16,23]$.

\section{Preliminaries}

Let $(X, d)$ and $Z$ be locally convex Hausdorff topological vector spaces, where $d$ is a metric which is compatible with the topology of $X$. Throughout this paper, suppose $C: X \times$ $Z \rightarrow 2^{Z}$ is a set-valued mapping such that, for any $x \in$ $X, C(x)$ is a pointed, closed, and convex cone in $Z$ with nonempty interior int $C(x)$, where $C(x)$ depends on $x$. We also assume that $e: X \rightarrow Z$ is a continuous vector-valued mapping and satisfies that, for any $x \in X, e(x) \in \operatorname{int} C(x)$.
Let $f: X \times X \times Z \rightarrow Z$ be a vector-valued mapping and $K_{i}: X \times Z \rightarrow 2^{X}, i=1,2$. The constraints appear in this paper are solution sets of the following (parametric) vector quasi-equilibrium problem, for each $z \in Z$ :

$$
\begin{array}{r}
\left(\operatorname{VQEP}_{z}\right) \text { find } \bar{x} \in K_{1}(\bar{x}, z) \text { s.t. } f(\bar{x}, y, z) \notin-\operatorname{int} C(\bar{x}), \\
\forall y \in K_{2}(\bar{x}, z) .
\end{array}
$$

Instead of writing $\left\{\left(\operatorname{VQEP}_{z}\right) \mid z \in Z\right\}$ for the family of quasiequilibrium problems, that is, the parametric problem, we will simply write (VQEP) in the sequel. Let $S: Z \rightarrow 2^{X}$ be the solution map of (VQEP). Let $Y=X \times Z, F: Y \times Y \rightarrow Z$, and $g: X \times Z \rightarrow Z$ be two functions with $g(x, z) \in D$. We consider the following bilevel vector equilibrium problem with equilibrium constraints:

$$
\begin{aligned}
& \text { (BVEPEC) finding } \bar{y} \in \operatorname{grS} \text { s.t., } \\
& F(\bar{y}, y) \notin-\operatorname{int} C(\bar{y}), \quad \forall y \in \operatorname{gr} S,
\end{aligned}
$$

where grS denotes the graph of $S$; that is, $\operatorname{gr} S:=\{(x, z) \mid x \in$ $S(z)\}$. We denote by $\Omega$ the set of solutions of (BVEPEC).

We first defined LP well-posedness notions.

Definition 1. A sequence $\left\{x_{n}^{*}\right\}=\left\{\left(x_{n}, z_{n}\right)\right\} \subset X \times Z$ is called type I LP approximating sequence for (BVEPEC) if and only if there exists a sequence of nonnegative real number $\left\{\epsilon_{n}\right\}$ with $\epsilon_{n} \rightarrow 0$ such that

$$
\begin{gathered}
d\left(x_{n}^{*}, \operatorname{gr} S\right) \leq \epsilon_{n} ; \\
F\left(x_{n}^{*}, y^{*}\right)+\epsilon_{n} e\left(x_{n}^{*}\right) \notin-\operatorname{int} C\left(x_{n}^{*}\right) \\
\forall y \in \operatorname{grS}(z), \quad z \in Z, \quad \text { where } y^{*}=(y, z) ;
\end{gathered}
$$

$\left\{x_{n}\right\}$ is an approximating sequence for the parametric problem (VQEP) corresponding to $\left\{z_{n}\right\}$.

Definition 2. A sequence $\left\{x_{n}^{*}\right\}=\left\{\left(x_{n}, z_{n}\right)\right\} \subset X \times Z$ is called type II LP approximating sequence for (BVEPEC) if and only if there exists a sequence of nonnegative real number $\left\{\epsilon_{n}\right\}$ with $\epsilon_{n} \rightarrow 0$ such that (3)-(5) hold and for any $n \in \mathbb{N}$ there exists $\left\{y_{n}^{*}\right\} \in \operatorname{grS}$ such that

$$
F\left(x_{n}^{*}, y_{n}^{*}\right)-\epsilon_{n} e\left(x_{n}\right) \in-C\left(x_{n}\right) .
$$

Definition 3. Problems (BVEPEC) is called type I (resp., type II) LP well-posed if and only if

(i) the solution set of (BVEPEC) is nonempty;

(ii) for any type I (resp., type II), LP approximating sequence of (BVEPEC) has a subsequence converging to a solution.

Recall now some notions. Let $X$ and $Z$ be as above and let $T: X \rightarrow 2^{Z}$ be a multifunction. $T$ is called lower semicontinuous (lsc) at $x_{0}$ if and only if $T\left(x_{0}\right) \cap U \neq \emptyset$. 
For some open subsets, $U \subseteq Z$ implies the existence of a neighborhood $N$ of $x_{0}$ such that $T(x) \cap U \neq \emptyset$ for all $x \in N$. T is upper semicontinuous (usc) at $x_{0}$ if and only if, for each open subset $U \supseteq T\left(x_{0}\right)$, there is a neighborhood $N$ of $x_{0}$ such that $U \supseteq T(N)$. T is called closed at $x_{0}$ if and only if, for each net $\left(x_{\alpha}, y_{\alpha}\right) \in \operatorname{grT}$ with $\left(x_{\alpha}, y_{\alpha}\right) \rightarrow\left(x_{0}, y_{0}\right)$, one has $y_{0} \in T\left(x_{0}\right)$. We say that $T$ satisfies a certain property in a subset $A \subseteq X$ if and only if $T$ satisfies it at every point of $A$. If $A=\operatorname{dom} T:=\{x \mid T(x) \doteq \emptyset\}$, we omit "in dom $T$ " in the saying. The following assertions are known:

(i) $T$ is lsc at $x_{0}$ if and only if $\forall x_{\alpha} \rightarrow x_{0}, \forall y \in T\left(x_{0}\right)$, $\exists y_{\alpha} \in T\left(x_{\alpha}\right)$, and $y_{\alpha} \rightarrow y$;

(ii) $T$ is closed if and only if grT is closed;

(iii) $T$ is usc at $x_{0}$ if $T(A)$ is compact for any compact subset $A$ of $\operatorname{dom} T$ and $T$ is closed at $x_{0}$;

(iv) $T$ is usc at $x_{0}$ if $Z$ is compact and $T$ is closed at $x_{0}$.

Definition 4 (see [23]). Let $X$ and $Y$ be topological spaces, and $f: X \rightarrow \overline{\mathbb{R}}$.

(i) $f$ is called upper 0 -level closed at $x_{0} \in X$ if and only if, for any sequence $\left\{x_{n}\right\}$ converging to $x_{0}$,

$$
\left[f\left(x_{n}\right) \geq 0, \forall n\right] \Longrightarrow\left[f\left(x_{0}\right) \geq 0\right] \text {. }
$$

(ii) $f$ is called lower 0 -level closed at $x_{0} \in X$ if and only if, for any sequence $\left\{x_{n}\right\}$ converging to $x_{0}$,

$$
\left[f\left(x_{n}\right) \leq 0, \forall n\right] \Longrightarrow\left[f\left(x_{0}\right) \leq 0\right] .
$$

Definition 5. Let $X$ be Hausdorff topological spaces and let $Z$ be a topological vector space $f: X \rightarrow Z$. The function $f$ is called upper 0 -level closed at $x_{0} \in X \times Y$ if and only if, for any $\left\{x_{n}\right\}$ converging to $x_{0}$,

$$
\left[f\left(x_{n}\right) \notin-\operatorname{int} C, \forall n\right] \Longrightarrow\left[f\left(x_{0}\right) \notin-\operatorname{int} C\right] \text {. }
$$

Definition 6 (see [32]). Let $A, B$ be nonempty subsets of metric space $X$. The Hausdorff distance $\mathscr{H}(\cdot, \cdot)$ between $A$ and $B$ is defined by

$$
\mathscr{H}(A, B)=\max \{\delta(A, B), \delta(B, A)\},
$$

where $\delta(A, B)=\sup _{a \in A} d(a, B)$ with $d(a, B)=\inf _{b \in B} d(a, b)$. Let $\left\{A_{n}\right\}$ be a sequence of nonempty subsets of $X$. We say that $\left\{A_{n}\right\}$ converges to $A$ in the sense of Hausdorff metric if $\mathscr{H}\left(A_{n}, A\right) \rightarrow 0$. It is easy to see that $\delta\left(A_{n}, A\right) \rightarrow 0$ if and only if $d\left(a_{n}, A\right) \rightarrow 0$ for all selection $a_{n} \in A_{n}$. For more details on this topic, we refer the readers to [32].

\section{Bilevel Vector Equilibrium Problems with Equilibrium Constraints (BVEPEC)}

In this section, we give some criteria and characterizations for LP well-posedness of (BVEPEC) using noncompactness. Now, we need the following notions of measures of noncompactness.
Definition 7. Let $M$ be a nonempty subset of a metric space $X$.

(i) The Kuratowski measure of $M$ is

$$
\begin{aligned}
& \mu(M) \\
& =\inf \left\{\epsilon>0 \mid M \subseteq \bigcup_{k=1}^{n} M_{k},\right. \\
& \left.\quad \operatorname{diam} M_{k} \leq \epsilon, k=1, \ldots, n \text {, for some } n \in \mathbb{N}\right\} .
\end{aligned}
$$

(ii) The Hausdorff measure of $M$ is

$$
\begin{array}{r}
\eta(M)=\inf \left\{\epsilon>0 \mid M \subseteq \bigcup_{k=1}^{n} B\left(x_{k}, \epsilon\right),\right. \\
\left.x_{k} \in X \text {, for some } n \in \mathbb{N}\right\} .
\end{array}
$$

(iii) The Istrătescu measure of $M$ is

$$
\begin{gathered}
\iota(M)=\inf \{\epsilon>0 \mid M \text { have no infinite } \\
\epsilon \text {-discrete subset }\} .
\end{gathered}
$$

The following inequalities are obtained in [33]:

$$
\eta(M) \leq \iota(M) \leq \mu(M) \leq 2 \eta(M)
$$

The measures $\mu, \eta$, and $\iota$ share many common properties and we will use $\gamma$ in the sequel to denote that either one of them $\gamma$ is a regular measure $[34,35]$; that is, it enjoys the following properties:

(i) $\gamma(M)=+\infty$ if and only if the set $M$ is unbounded;

(ii) $\gamma(M)=\gamma(\mathrm{cl} M)$;

(iii) if $\gamma(M)=0$, then $M$ is a totally bounded set;

(iv) if $X$ is a complete space and if $\left\{A_{n}\right\}$ is a sequence of closed subset of $X$ such that $A_{n+1} \subseteq A_{n}$ for each $n \in \mathbb{N}$ and $\lim _{n \rightarrow+\infty} \mathscr{H}\left(A_{n}, K\right)=0$, where $\mathscr{H}$ is the Hausdorff metric;

(v) if $M \subseteq N$, then $\gamma(M) \leq \gamma(N)$.

As above, $S(z)$ denotes the solution set of $\left(\mathrm{VQEP}_{z}\right)$. For positive $\epsilon$, the $\epsilon$-solution set of $\left(\mathrm{VQEP}_{z}\right)$ is defined by

$$
\begin{gathered}
\widetilde{S}(z, \epsilon)=\left\{x \in K_{1}(x, z) \mid f(x, y, z)+\epsilon e(x) \notin-\operatorname{int} C(x),\right. \\
\left.\forall y \in K_{2}(x, z)\right\} .
\end{gathered}
$$


For positive $\xi$ and $\epsilon$, the corresponding approximate solution sets for (BVEPEC) are defined, respectively, by

$$
\begin{aligned}
& \Gamma_{1}(\xi, \epsilon):=\left\{x^{*}=(x, z) \in K_{1}(x, z) \times Z \mid d\left(x^{*}, \operatorname{gr} S\right) \leq \epsilon,\right. \\
& F\left(x^{*}, y^{*}\right)+\epsilon e(x) \notin-\operatorname{int} C(x), \forall y^{*} \in \operatorname{gr} S, \\
& f(x, y, z)+\xi e(x) \notin-\operatorname{int} C(x), \\
&\left.\forall y \in K_{2}(x, z)\right\} \\
& \Gamma_{2}(\xi, \epsilon):=\left\{x^{*}=(x, z) \in K_{1}(x, z) \times Z \mid d\left(x^{*}, \operatorname{gr} S\right) \leq \epsilon,\right. \\
& F\left(x^{*}, y^{*}\right)+\epsilon e(x) \notin-\operatorname{int} C(x), \forall y^{*} \in \operatorname{gr} S, \\
& f(x, y, z)+\xi e(x) \notin-\operatorname{int} C(x), \\
&\left.\forall y \in K_{2}(x, z), F\left(x^{*}, y^{*}\right)-\epsilon e(x) \in-C(x)\right\} .
\end{aligned}
$$

In terms of a measure $\gamma \in\{\mu, \eta, \imath\}$ of noncompactness we have the following result.

Theorem 8. Let $X$ and $Z$ be complete and $\gamma \in\{\mu, \eta, \iota\}$. Assume that

(i) in $X \times Z, K_{1}$ is closed and $K_{2}$ is lsc;

(ii) $f$ is upper 0 -level closed in $K_{1}(X, Z) \times K_{2}(X, Z) \times Z$;

(iii) $F\left(\cdot, y^{*}\right)$ is upper 0-level closed in $X \times Z$, for all $y^{*} \in$ grS;

(iv) the mapping $W: X \times Z \rightarrow 2^{Z}$ defined by $W(x)=$ $Z \backslash-\operatorname{int} C(x)$ is closed.

Then, (BVEPEC) is type I LP well posed if and only if

$$
\begin{array}{r}
\gamma\left(\Gamma_{1}(\xi, \epsilon)\right) \longrightarrow 0 \quad\left(\text { resp., } \gamma\left(\Gamma_{3}(\xi, \epsilon)\right) \longrightarrow 0\right) \longrightarrow 0 \\
\text { as }(\xi, \epsilon) \longrightarrow(0,0) .
\end{array}
$$

Proof. By the relationship (14), the proof is similar for the three mentioned measures of noncompactness. We discuss only the case $\gamma=\mu$, the Kuratowski measure. Assume that (BVEPEC) is type I LP well posed. The solution set $\Omega$ of (BVEPEC) clearly the relation $\Omega \subseteq \Gamma_{1}(\xi, \epsilon)$. Hence,

$$
\begin{aligned}
\mathscr{H}\left(\Gamma_{1}(\xi, \epsilon), \Omega\right) & =\max \left\{\delta\left(\Gamma_{1}(\xi, \epsilon), \Omega\right), \delta\left(\Omega, \Gamma_{1}(\xi, \epsilon)\right)\right\} \\
& =\delta\left(\Gamma_{1}(\xi, \epsilon), \Omega\right) .
\end{aligned}
$$

Let $\left\{x_{N}^{*}\right\}=\left\{\left(x_{n}, z_{n}\right)\right\}$ be in $\Omega$. Since $\left\{x_{n}^{*}\right\}$ is an approximating sequence, it has a subsequence converging to some points of $\Omega$. Therefore, $\Omega$ is compact.

Assume that $\Omega \subseteq \bigcup_{k=1}^{n} M_{k}$ with $\operatorname{diam} M_{k} \leq \epsilon, \forall k=$ $1, \ldots, n$. Setting $N_{k}=\left\{z \in X \mid d\left(z, M_{k}\right) \leq \mathscr{H}\left(\Gamma_{1}(\xi, \epsilon), \Omega\right)\right\}$, it obvious that $\Gamma_{1}(\xi, \epsilon) \subseteq \bigcup_{k=1}^{n} N_{k}$ and $\operatorname{diam} N_{k} \leq \epsilon+$ $2 \mathscr{H}\left(\Gamma_{1}(\xi, \epsilon), \Omega\right)$. Since $\Omega$ is compact and $\mu(\Omega)=0$, then we get

$$
\mu\left(\Gamma_{1}(\xi, \epsilon)\right) \leq 2 \mathscr{H}\left(\Gamma_{1}(\xi, \epsilon), \Omega\right)+\mu(\Omega)=2 \delta\left(\Gamma_{1}(\xi, \epsilon), \Omega\right) .
$$

Next, we show that $\mathscr{H}\left(\Gamma_{1}(\xi, \epsilon), \Omega\right) \rightarrow 0$ as $(\xi, \epsilon) \rightarrow(0,0)$. By contradiction, suppose the existence of $\rho>0,(\xi, \epsilon) \rightarrow(0,0)$ and $x_{n} \in \Gamma_{1}\left(\xi_{n}, \epsilon_{n}\right)$ such that $d\left(x_{n}, \Omega\right) \geq \rho, \forall n \in \mathbb{N}$. This contradicts the type I LP well-posedness. So, $\mathscr{H}\left(\Gamma_{1}(\xi, \epsilon), \Omega\right) \rightarrow 0$ as $(\xi, \epsilon) \rightarrow(0,0)$. It follows that (17) holds.

Conversely, first, we show that for all $\xi, \epsilon>0, \Gamma_{1}(\xi, \epsilon)$ is closed. Assume that $\mu\left(\Gamma_{1}(\xi, \epsilon)\right) \rightarrow 0$ as $(\xi, \epsilon) \rightarrow(0,0)$. Let $\left\{x_{n}^{*}\right\}=\left\{\left(x_{n}, z_{n}\right)\right\} \subset \Gamma_{1}(\xi, \epsilon)$ with $\left\{x_{n}^{*}\right\} \rightarrow x^{*}:=(x, z)$. Then, for all $y^{*} \in$ grS and $y_{n} \in K_{2}\left(x_{n}, z_{n}\right)$,

$$
\begin{gathered}
d\left(x_{n}^{*}, \mathrm{gr} S\right) \leq \epsilon ; \\
F\left(x_{n}^{*}, y^{*}\right)+\epsilon e\left(x_{n}^{*}\right) \notin-\operatorname{int} C\left(x_{n}^{*}\right), \\
f\left(x_{n}, y_{n}, z_{n}\right)+\xi e\left(x_{n}\right) \notin-\operatorname{int} C\left(x_{n}\right) .
\end{gathered}
$$

As $K_{1}$ is closed at $(x, z)$, we have $x \in K_{1}(x, z)$. From (20), we obtain that $d\left(x^{*}\right.$, grS $) \leq \epsilon$. By the upper 0 -level closedness of $F$ in first argument and assumption (iv), one obtain

$$
F\left(x^{*}, y^{*}\right)+\epsilon e\left(x^{*}\right) \in W\left(x^{*}\right)
$$

that is,

$$
F\left(x^{*}, y^{*}\right)+\epsilon e\left(x^{*}\right) \notin-\operatorname{int} C\left(x^{*}\right), \quad \forall y^{*} \in \operatorname{grS} .
$$

Next, we show by contrapositive that $f(x, y, z)+\xi e(x) \notin$ - int $C(x), \forall y \in K_{2}(x, z)$. Suppose that there exist $y \in$ $K_{2}(x, z)$ such that $f(x, y, z)+\xi e(x) \in-\operatorname{int} C(x)$. Since $K_{2}$ is lsc at $(x, z)$, there exist $y_{n} \in K_{2}(x, z)$ such that $y_{n} \rightarrow y$. By upper 0-level closedness of $f$ at $(x, y, z)$, there is $n_{0} \in \mathbb{N}$ such that

$$
f\left(x_{n}, y_{n}, z_{n}\right)+\xi e\left(x_{n}\right) \in-\operatorname{int} C\left(x_{n}\right), \quad \forall n \geq n_{0} .
$$

That is a contradiction. Thus, we have

$$
f(x, y, z)+\xi_{e}(x) \notin-\operatorname{int} C(x), \quad \forall y \in K_{2}(x, z) .
$$

Therefore, $x^{*} \in \Gamma_{1}(\xi, \epsilon)$ and so $\Gamma_{1}(\xi, \epsilon)$ is closed.

Secondly, we show that

$$
\Omega=\bigcap_{\xi, \epsilon>0} \Gamma_{1}(\xi, \epsilon)
$$

It is obvious that $\Omega \subset \bigcap_{\xi, \epsilon>0} \Gamma_{1}(\xi, \epsilon)$. Now, suppose that $\left(\xi_{n}, \epsilon_{n}\right) \rightarrow(0,0)$ and $x^{*} \in \bigcap_{\xi, \epsilon>0} \Gamma_{1}\left(\xi_{n}, \epsilon_{n}\right)$. Thus, we have

$$
\begin{gathered}
d\left(x^{*}, \operatorname{gr} S\right) \leq \epsilon_{n} ; \\
F\left(x^{*}, y^{*}\right)+\epsilon_{n} e\left(x^{*}\right) \notin-\operatorname{int} C\left(x^{*}\right), \quad \forall y^{*} \in \operatorname{gr} S, \\
f(x, y, z)+\xi_{n} e(x) \notin-\operatorname{int} C(x), \quad \forall y \in K_{2}(x, z) .
\end{gathered}
$$

By (27) and (28) and closedness of $W\left(x^{*}\right)$, we obtain

$$
\begin{gathered}
F\left(x^{*}, y^{*}\right) \in W\left(x^{*}\right), \quad \forall y^{*} \in \operatorname{gr} S, \\
f(x, y, z) \in W(x), \quad \forall y \in K_{2}(x, z) .
\end{gathered}
$$

That is, $x^{*} \in \Gamma_{1}(\xi, \epsilon)$. Hence, (25) holds. 
We know that $\mu\left(\Gamma_{1}(\xi, \epsilon)\right) \rightarrow 0$ as $(\xi, \epsilon) \rightarrow(0,0)$. Then, by properties of $\mu$, we see that $\Omega$ is compact and $\mathscr{H}\left(\Gamma_{1}(\xi, \epsilon)\right) \rightarrow$ 0 as $(\xi, \epsilon) \rightarrow(0,0)$. Let $\left\{x_{n}^{*}\right\}=\left\{\left(x_{n}, z_{n}\right)\right\}$ be a type I LP approximating solution sequence for (BVEPEC). There is $\left\{\left(\xi_{n}, \epsilon_{n}\right)\right\} \rightarrow(0,0)$ such that, for all $y^{*} \in \operatorname{grS}$ and $y \in$ $K_{2}(x, z)$,

$$
\begin{gathered}
d\left(x_{n}^{*}, \operatorname{gr} S\right) \leq \epsilon_{n} \\
F\left(x_{n}^{*}, y^{*}\right)+\epsilon_{n} e\left(x_{n}^{*}\right) \notin-\operatorname{int} C\left(x_{n}^{*}\right), \\
f\left(x_{n}, y, z_{n}\right)+\xi_{n} e\left(x_{n}\right) \notin-\operatorname{int} C\left(x_{n}\right) .
\end{gathered}
$$

Therefore, $x_{n}^{*} \in \Gamma_{1}\left(\xi_{n}, \epsilon_{n}\right)$. It follows that, from $\mu\left(\Gamma_{1}(\xi, \epsilon)\right) \rightarrow$ 0 as $(\xi, \epsilon) \rightarrow(0,0)$,

$$
d\left(x_{n}^{*}, \Omega\right) \leq \mathscr{H}\left(\Gamma_{1}(\xi, \epsilon), \Omega\right) \longrightarrow 0 .
$$

By the compactness of $\Omega$, there is a subsequence of $\left\{x_{n}\right\}$ convergent to some points of $\Omega$. Hence, (BVEPEC) is type I LP well posed. This completes the proof.

Similar to Theorem 8 , we can prove that the following results.

Theorem 9. Let $X$ and $Z$ be complete and $\gamma \in\{\mu, \eta, \iota\}$. Assume that

(i) in $X \times Z, K_{1}$ is closed and $K_{2}$ is lsc;

(ii) $f$ is upper 0-level closed in $K_{1}(X, Z) \times K_{2}(X, Z) \times Z$;

(iii) $F\left(\cdot, y^{*}\right)$ is upper 0-level closed in $X \times Z$, for all $y^{*} \in$ grS;

(iv) the mapping $W: X \times Z \rightarrow 2^{Z}$ defined by $W(x)=$ $Z \backslash$ - int $C(x)$ is closed;

(v) the set-valued mapping $C: X \times Z \rightarrow 2^{Z}$ is closed;

(vi) for any $x \in \Omega, F\left(x^{*}, y^{*}\right) \in-\partial C$ and $\exists y^{*} \in \operatorname{grS}$ and for any $x \in S, f(x, y, z) \in-\partial C$ and $\exists y \in K_{2}(x, z)$.

Then, (BVEPEC) is type II LP well posed if and only if $\gamma\left(\Gamma_{2}(\xi, \epsilon)\right) \rightarrow 0\left(\right.$ resp., $\left.\gamma\left(\Gamma_{4}(\xi, \epsilon)\right) \rightarrow 0\right) \rightarrow 0$ as $(\xi, \epsilon) \rightarrow$ $(0,0)$.

\section{Optimization Problem with Equilibrium Constraints (OPEC)}

In this section, we will present the criteria and characterization for four types of (BVEPEC) and introduce a gap function for (BVEPEC) using the nonlinear scalarization function and then we investigate the equivalent relations between the LP well-posedness for bilevel vector optimization problem with equilibrium constraints (BVOPEC) and the LP wellposedness for vector equilibrium problem with equilibrium constraints (BVEPEC). Now, consider the following optimization problem with equilibrium constraints.

Let $S: Z \rightarrow 2^{X}$ be the solution map of (VQEP). Let $\phi: X \times Z \rightarrow R$, where $R=(-\infty,+\infty]$. The bilevel vector optimization problem with equilibrium constraints is as follows:

(BVOPEC) minimize $\phi(x, z)$ s.t., $x^{*}:=(x, z) \in$ grS.
Note that $z$ is a parameter of the vector quasi-equilibrium problem defining the constraint, but it is a component of the decision variable $(x, z)$ of (BVEPEC) and (BVOPEC) and these problems are not parametric.

Definition 10. A sequence $\left\{x_{n}^{*}\right\}=\left\{\left(x_{n}, z_{n}\right)\right\} \subset X \times Z$ is called type I LP minimizing sequence for (BVOPEC) if and only if

$$
\begin{gathered}
d\left(x_{n}^{*}, \operatorname{gr} S\right) \longrightarrow 0 ; \\
\limsup _{n \rightarrow \infty} \phi\left(x_{n}^{*}\right) \leq v^{*} ;
\end{gathered}
$$

$\left\{x_{n}\right\}$ is an approximating sequence for (QEP)

corresponding to $\left\{z_{n}\right\}$.

Definition 11. A sequence $\left\{x_{n}^{*}\right\}=\left\{\left(x_{n}, z_{n}\right)\right\} \subset X \times Z$ is called type II LP minimizing sequence for (BVOPEC) if and only if (33) and (35) hold and

$$
\lim _{n \rightarrow \infty} \phi\left(x_{n}^{*}\right)=v^{*}
$$

Definition 12. Problems (BVOPEC) is called type I (resp., type II) LP well posed if and only if

(i) the solution set of (BVOPEC) is nonempty;

(ii) for any type I (resp., type II), LP minimizing sequence of (BVOPEC) has a subsequence converging to a solution.

Now, we recall the definition of nonlinear scalarization function introduced by Chen et al. [36].

The nonlinear scalarization function $\xi_{e}: Z \rightarrow \mathbb{R}$ is defined by

$$
\xi_{e}(x)=\min \{\lambda \in \mathbb{R}: x-\lambda e(x) \in-C(x)\} .
$$

Definition 13. A mapping $\phi: Y \rightarrow \mathbb{R} \cup\{+\infty\}$ is called a gap function for (BVEPEC) if

(i) $\phi(x) \geq 0, \forall x \in Y$;

(ii) $\phi\left(x^{*}\right)=0, \forall x^{*} \in \operatorname{grS}$ if and only if $x^{*} \in \Omega$.

We introduced the following gap function defined by

$$
\phi^{*}(x)=\sup _{y \in \operatorname{grS}}\left\{-\xi_{e}(F(x, y))\right\}, \quad \forall x \in \operatorname{grS} .
$$

Remark 14. (i) By Definition 4, it is easy to see that $\phi^{*}(x)$ is a gap function for (EPEC). Moreover, if $\Omega \neq \emptyset$, then $\operatorname{Dom}\left(\phi^{*}(x)\right) \cap \operatorname{gr} S \neq \emptyset$.

(ii) By Definition 4 , it is clear that $x_{0} \in \Omega$ if and only if $x_{0}$ minimizes $\phi^{*}(x)$ over grS with $\phi^{*}\left(x_{0}\right)=0$.

Now, we prove the following lemma.

Lemma 15. Let for any $x \in \operatorname{grS}, F(x, x) \in-\partial C(x)$, where $\partial C(x)$ is the topological boundary of $C(x)$ and $F(\cdot, y)$ is upper 0 -level closed on $X \times Z$, for all $y \in$ grS. Then, the mapping $\phi^{*}$ defined by (38) is lower 0 -level closed on $X \times Z$. 
Proof. Suppose that $\left\{x_{n}^{*}\right\} \in \operatorname{grS}$ satisfies $x_{n}^{*} \rightarrow x^{*} \in \operatorname{grS}$ and $\phi^{*}\left(x_{n}^{*}\right) \leq b, \forall n \in \mathbb{N}$. Follows from (38)

$$
\phi^{*}\left(x_{n}\right)^{*}=\sup _{y \in \operatorname{grS}}\left\{-\xi_{e}\left(F\left(x_{n}^{*}, y\right)\right)\right\} \leq 0 .
$$

Then, $\xi_{e}\left(F\left(x_{n}^{*}, y\right)\right) \geq 0, \forall y \in$ grS. By the upper closed 0 -level of $F$ in first argument, we know that

$$
\xi_{e} F\left(x^{*}, y\right) \geq 0, \quad \forall y \in \text { grS. }
$$

That is,

$$
\phi^{*}\left(x^{*}\right)=\sup _{y \in \operatorname{grS}}\left\{-\xi_{e}\left(F\left(x^{*}, y\right)\right)\right\} \leq 0 .
$$

Then, we have that $\phi^{*}\left(x^{*}\right)$ is lower closed 0 -level. This completes the proof.

Theorem 16. Suppose that the assumptions of Lemma 15 are satisfied. Then the following results hold:

(i) (BVEPEC) is the type I LP well-posedness if and only if (BVOPEC) is the type I LP well-posedness with the function $\phi$ defined by (38).

(ii) (BVEPEC) is the type II LP well-posedness if and only if (BVOPEC) is the type II LP well-posedness with the function $\phi$ defined by (38).

Proof. (i) We know that $\phi^{*}$ is a gap function of (BVEPEC), and $x^{*} \in \Omega$ if and only if $x^{*} \in$ grS with $\bar{v}=\phi^{*}\left(x^{*}\right)=0$. Assume that $\left\{x_{n}^{*}\right\}=\left\{\left(x_{n} \cdot z_{n}\right)\right\}$ is any type I LP approximating solution sequence for (BVEPEC). Then, there exist $\left\{\epsilon_{n}\right\}>0$ with $\epsilon_{n} \rightarrow 0$ such that

$$
\begin{aligned}
d\left(x_{n}^{*}, \operatorname{gr} S\right) \leq \epsilon_{n} ; & \\
F\left(x_{n}^{*}, y^{*}\right)+\epsilon e\left(x_{n}^{*}\right) \notin-\operatorname{int} C\left(x_{n}^{*}\right), & \forall y^{*} \in \operatorname{gr} S, \\
f\left(x_{n}, y_{n}, z_{n}\right)+\xi e\left(x_{n}\right) \notin-\operatorname{int} C\left(x_{n}\right), & \forall y_{n} \in K_{2}\left(x_{n}, z_{n}\right) .
\end{aligned}
$$

It follows from (43) that

$$
\xi_{e}\left(F\left(x_{n}^{*}, y^{*}\right)\right) \geq-\epsilon_{n}, \quad \forall y^{*} \in \operatorname{grS} .
$$

Then, we obtain

$$
\phi\left(x_{n}^{*}\right)=\sup \left\{-\xi_{e}\left(F\left(x_{n}^{*}, y^{*}\right)\right)\right\} \leq \epsilon_{n}, \quad \forall y^{*} \in \operatorname{grS} .
$$

Hence,

$$
\limsup _{n \rightarrow \infty} \phi\left(x_{n}^{*}\right) \leq 0, \quad \text { since } \epsilon_{n} \longrightarrow 0 .
$$

Therefore, $\left\{x_{n}\right\}$ is a type I LP minimizing sequence for (BVOPEC).

Conversely, assume that $\left\{x_{n}^{*}\right\}$ is any type I LP minimizing sequence for (BVOPEC). Then, $d\left(x_{n}^{*}, \operatorname{gr} S\right) \rightarrow 0$, $\limsup _{n \rightarrow \infty} \phi\left(x_{n}^{*}\right) \leq 0$, and $f\left(x_{n}, y_{n}, z_{n}\right)+\xi_{n} e\left(x_{n}\right) \notin$ $-\operatorname{int} C\left(x_{n}\right), \forall y_{n} \in K_{2}(x, z)$. Then, there exist $\left\{\epsilon_{n}\right\}>0$ with $\epsilon_{n} \rightarrow 0$ satisfying $d\left(x_{n}^{*}, \operatorname{gr} S\right) \leq \epsilon_{n}$;

$$
\phi\left(x_{n}^{*}\right)=\sup \left\{-\xi_{e}\left(F\left(x_{n}^{*}, y^{*}\right)\right)\right\} \leq \epsilon_{n}, \quad \forall y^{*} \in \operatorname{grS} .
$$

Then, we get $\xi_{e}\left(F\left(x_{n}^{*}, y^{*}\right)\right) \geq-\epsilon_{n}$ or, equivalently,

$$
F\left(x_{n}^{*}, y^{*}\right)+\epsilon_{n} e\left(x_{n}^{*}\right) \notin-\operatorname{int} C\left(x_{n}^{*}\right), \quad \forall y \in \operatorname{grS} .
$$

Hence, $\left\{x_{n}^{*}\right\}$ is a type I LP approximating solution sequence for (BVEPEC). It follows that (BVEPEC) is the type I LP wellposedness if and only if (BVOPEC) is the type I LP wellposedness with the function $\phi$.

The proof of (ii) is similar to (i) and we are omitted. This completes the proof.

\section{Conflict of Interests}

The authors declare that there is no conflict of interests regarding the publication of this paper.

\section{Acknowledgments}

The authors would like to thank The Commission on Higher Education for financial support. Moreover, Kamonrat Sombut is also supported by The Strategic Scholarships Fellowships Frontier Research Networks under Grant no. CHEPh.D-THA-SUP/86/2550, Thailand.

\section{References}

[1] A. N. Tikhonov, "On the stability of the functional optimization problem," USSR Computational Mathematics and Mathematical Physics, vol. 6, no. 4, pp. 28-33, 1966.

[2] E. S. Levitin and B. T. Polyak, "Convergence of minimizing sequences in conditional extremum problems," Soviet Mathematics Doklady, vol. 7, pp. 764-767, 1966.

[3] Y.-P. Fang and R. Hu, "Parametric well-posedness for variational inequalities defined by bifunctions," Computers \& Mathematics with Applications, vol. 53, no. 8, pp. 1306-1316, 2007.

[4] M. B. Lignola and J. Morgan, "Well-posedness for optimization problems with constraints defined by variational inequalities having a unique solution," Journal of Global Optimization, vol. 16, no. 1, pp. 57-67, 2000.

[5] M. B. Lignola, "Well-posedness and $L$-well-posedness for quasivariational inequalities," Journal of Optimization Theory and Applications, vol. 128, no. 1, pp. 119-138, 2006.

[6] R. Lucchetti, Recent Developments in Well-Posed Variational Problems, Kluwer Academic, Dordrecht, The Netherlands, 1995.

[7] R. Lucchetti and F. Patrone, "A characterization of Tyhonov well-posedness for minimum problems, with applications to variational inequalities," Numerical Functional Analysis and Optimization, vol. 3, no. 4, pp. 461-476, 1981.

[8] R. Lucchetti and F. Patrone, "Hadamard and Tyhonov wellposedness of a certain class of convex functions," Journal of Mathematical Analysis and Applications, vol. 88, no. 1, pp. 204215, 1982.

[9] M. Margiocco, F. Patrone, and L. Pusillo Chicco, "A new approach to Tikhonov well-posedness for Nash equilibria," Optimization, vol. 40, no. 4, pp. 385-400, 1997.

[10] M. Margiocco, F. Patrone, and L. Pusillo Chicco, "Metric characterizations of Tikhonov well-posedness in value," Journal of Optimization Theory and Applications, vol. 100, no. 2, pp. 377$387,1999$. 
[11] M. Margiocco, F. Patrone, and L. Pusillo, "On the Tikhonov well-posedness of concave games and Cournot oligopoly games," Journal of Optimization Theory and Applications, vol. 112, no. 2, pp. 361-379, 2002.

[12] J. Morgan, "Approximations and well-posedness in multicriteria games," Annals of Operations Research, vol. 137, pp. 257-268, 2005.

[13] A. S. Konsulova and J. P. Revalski, "Constrained convex optimization problems-well-posedness and stability," Numerical Functional Analysis and Optimization, vol. 15, no. 7-8, pp. 889907, 1994.

[14] G. Beer and R. Lucchetti, “The epi-distance topology: continuity and stability results with applications to convex optimization problems," Mathematics of Operations Research, vol. 17, no. 3, pp. 715-726, 1992.

[15] X. X. Huang and X. Q. Yang, "Levitin-Polyak well-posedness of constrained vector optimization problems," Journal of Global Optimization, vol. 37, no. 2, pp. 287-304, 2007.

[16] S. J. Li and M. H. Li, "Levitin-Polyak well-posedness of vector equilibrium problems," Mathematical Methods of Operations Research, vol. 69, no. 1, pp. 125-140, 2009.

[17] N.-J. Huang, X.-J. Long, and C.-W. Zhao, "Well-posedness for vector quasi-equilibrium problems with applications," Journal of Industrial and Management Optimization, vol. 5, no. 2, pp. 341-349, 2009.

[18] M. H. Li, S. J. Li, and W. Y. Zhang, "Levitin-Polyak wellposedness of generalized vector quasi-equilibrium problems," Journal of Industrial and Management Optimization, vol. 5, no. 4, pp. 683-696, 2009.

[19] J. V. Outrata, "A generalized mathematical program with equilibrium constraints," SIAM Journal on Control and Optimization, vol. 38, no. 5, pp. 1623-1638, 2000.

[20] B. S. Mordukhovich, "Characterizations of linear suboptimality for mathematical programs with equilibrium constraints," Mathematical Programming, vol. 120, no. 1, pp. 261-283, 2009.

[21] M. B. Lignola and J. Morgan, " $\alpha$-well-posedness for Nash equilibria and for optimization problems with Nash equilibrium constraints," Journal of Global Optimization, vol. 36, no. 3, pp. 439-459, 2006.

[22] T. Q. Bao, P. Gupta, and B. S. Mordukhovich, "Necessary conditions in multiobjective optimization with equilibrium constraints," Journal of Optimization Theory and Applications, vol. 135, no. 2, pp. 179-203, 2007.

[23] L. Q. Anh, P. Q. Khanh, and D. T. M. Van, "Well-posedness under relaxed semicontinuity for bilevel equilibrium and optimization problems with equilibrium constraints," Journal of Optimization Theory and Applications, vol. 153, no. 1, pp. 42-59, 2012.

[24] Q. H. Ansari, "Vector equilibrium problems and vector variational inequalities," in Vector Variational Inequalities and Vector Equilibria, F. Giannessi, Ed., Mathematical Theories, p. 116, Kluwer, Dordrecht, The Netherlands, 2000.

[25] F. Giannessi, Ed., Vector Variational Inequalities and Vector Equilibria, vol. 38 of Nonconvex Optimization and its Applications, Kluwer Academic, Dordrecht, The Netherlands, 2000.

[26] G. Y. Chen, X. Q. Yang, and H. Yu, "A nonlinear scalarization function and generalized quasi-vector equilibrium problems," Journal of Global Optimization, vol. 32, no. 4, pp. 451-466, 2005.

[27] S. J. Li, K. L. Teo, and X. Q. Yang, "Generalized vector quasiequilibrium problems," Mathematical Methods of Operations Research, vol. 61, no. 3, pp. 385-397, 2005.
[28] S. J. Li, K. L. Teo, X. Q. Yang, and S. Y. Wu, "Gap functions and existence of solutions to generalized vector quasi-equilibrium problems," Journal of Global Optimization, vol. 34, no. 3, pp. 427-440, 2006.

[29] Q. H. Ansari and F. Flores-Bazán, "Generalized vector quasiequilibrium problems with applications," Journal of Mathematical Analysis and Applications, vol. 277, no. 1, pp. 246-256, 2003.

[30] M. Bianchi, N. Hadjisavvas, and S. Schaible, "Vector equilibrium problems with generalized monotone bifunctions," Journal of Optimization Theory and Applications, vol. 92, no. 3, pp. 527-542, 1997.

[31] W. Song, "On generalized vector equilibrium problems," Applied Mathematics Letters, vol. 12, pp. 53-56, 2002.

[32] C. Kuratowski, Topology. Panstwowe Wydawnicto Naukowa, vol. 1, Warszawa, Poland, 1958.

[33] J. Daneš, "On the Istrătescu measure of noncompactness," Bulletin Mathématique de la Société des Sciences Mathématiques de Roumanie, vol. 16, no. 4, pp. 403-406, 1972.

[34] J. Banaś and K. Goebel, Measures of Noncompactness in Banach Spaces, vol. 60 of Lecture Notes in Pure and Applied Mathematics, Marcel Dekker, New York, NY, USA, 1980.

[35] V. Rakočević, "Measures of noncompactness and some applications," Filomat, no. 12, pp. 87-120, 1998.

[36] G.-Y. Chen, X. Huang, and X. Yang, Vector Optimization, SetValued and Variational Analysis, vol. 541 of Lecture Notes in Economics and Mathematical Systems, Springer, Berlin, Germany, 2005. 


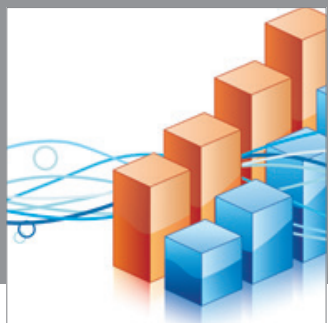

Advances in

Operations Research

mansans

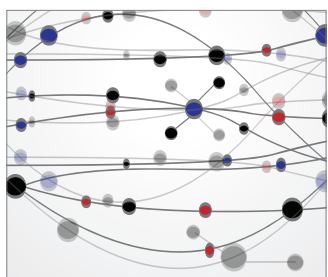

The Scientific World Journal
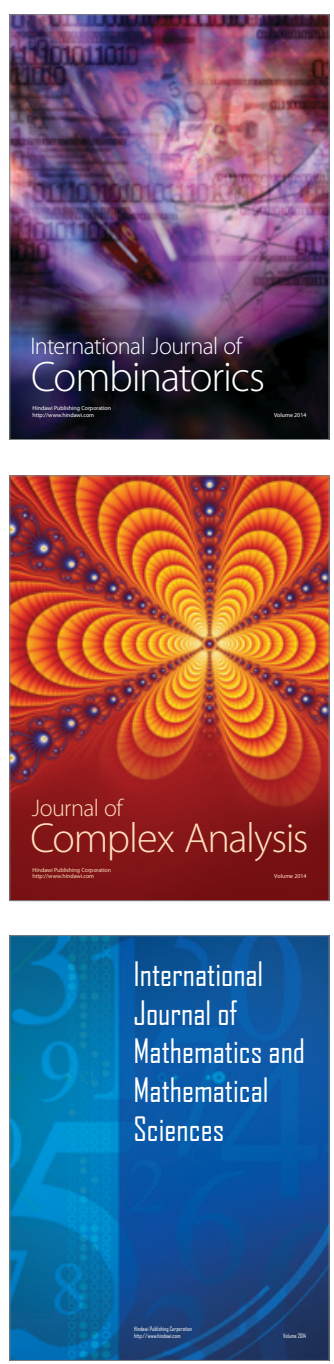
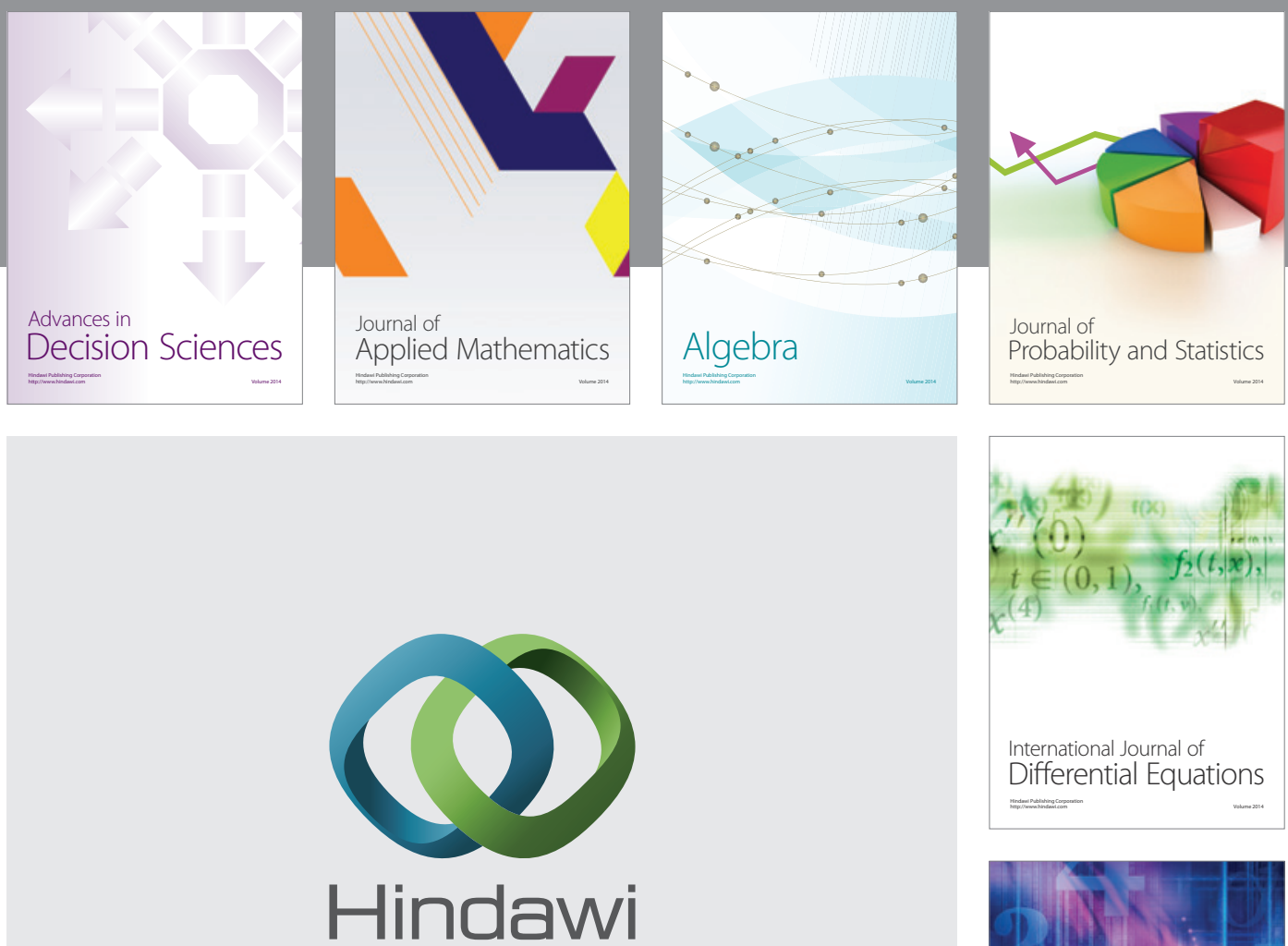

Submit your manuscripts at http://www.hindawi.com
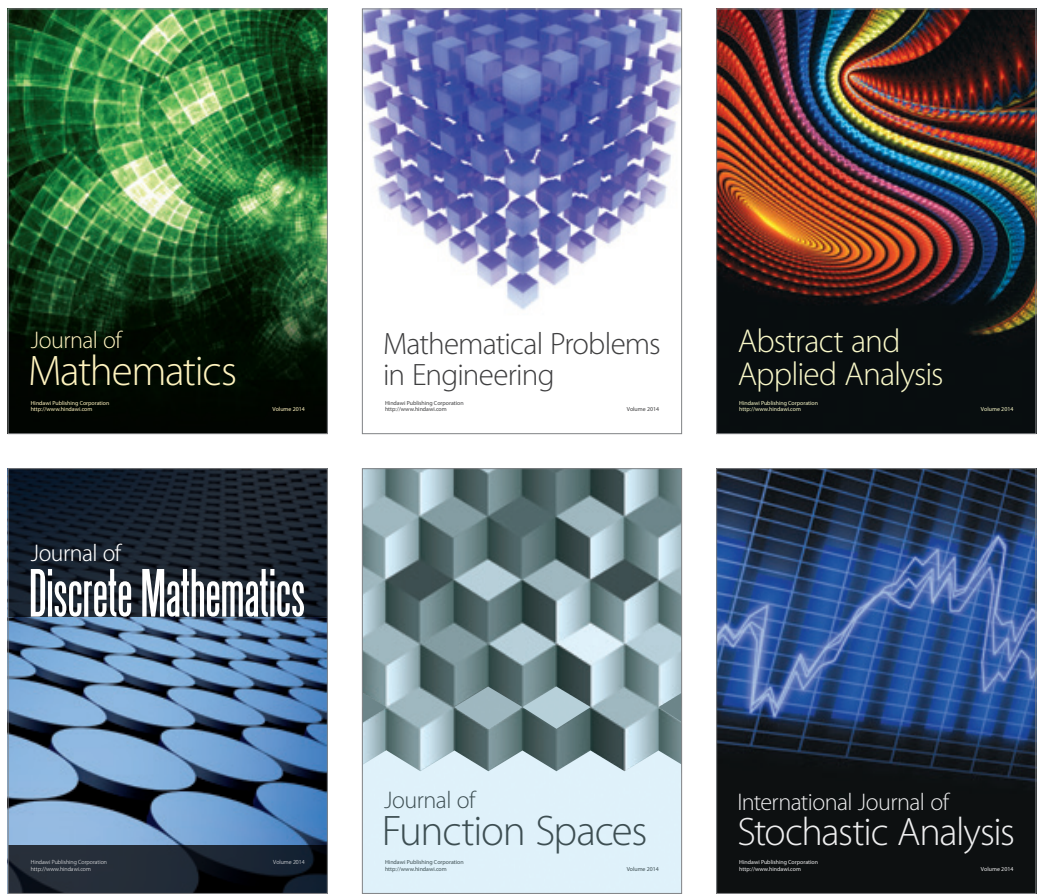

Journal of

Function Spaces

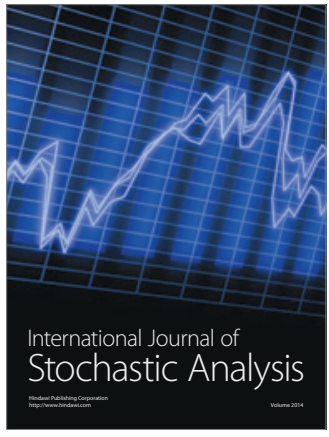

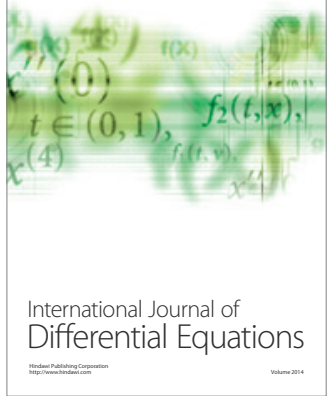
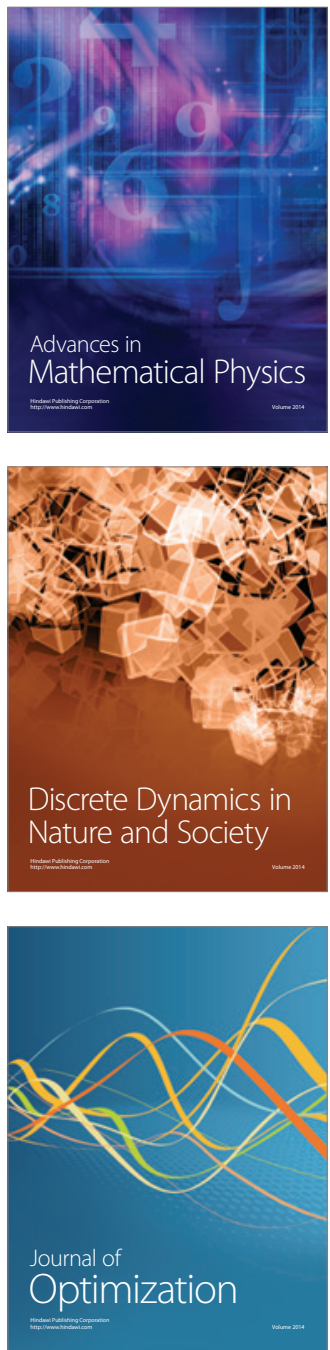